\title{
Das Johann Adolf Hasse zugeschriebene Passions-Oratorium La morte di Cristo und seine musikhistorische Einordnung
}

\section{The Passion Oratorio "La morte di Cristo" Attributed to Johann Adolf Hasse and Its Musical-Historical Context}

Steffen Voss / steffen.voss@bsb-muenchen.de

Bayerische Staatsbibliothek, München, DE

\begin{abstract}
The passion oratorio La morte di Cristo, attributed in two German sources from the late $18^{\text {th }}$ or early 19th century to Johann Adolf Hasse, was identified in 1976 by Reinhard Strohm as a sacred pasticcio, based on opera arias by Hasse and other composers of the Neapolitan school like Francesco Feo and Leonardo Leo. A comparison with surviving libretti shows that this pasticcio score is identical with the oratorio La vittima d'amore o sia la morte di Giesù Christo salvatore nostro, attributed to Josef Umstatt and performed 1741 in Brno and 1744 in Prague. Czech musicologists found out that one aria which hadn't been identified by Strohm, is a borrowing from Antonio Caldaras Viennese oratorio Morte e sepoltura di Cristo. A Berlin score of this work, which can be linked to a performance in Brno, has the words of the aria from La vittima d'amore written under the orginal text. Umstatt, who worked in Brno at the time when these performances took place, might have been the arranger of the pasticcio and composer of the sinfonia, the recitatives and the final chorus.
\end{abstract}

\section{Keywords}

Oratorium, Pasticcio, Brno, Johann Adolf Hasse, Francesco Feo, Leonardo Leo, Giovanni Porta, Antonio Caldara, Josef Umstatt 
Zu den ungewöhnlichsten apokryphen Werken Johann Adolf Hasses gehört ein italienisches Passionsoratorium, das unter dem Titel La morte di Cristo überliefert ist. Das Werk ist lediglich in zwei relativ späten Abschriften in München und Hamburg erhalten, die Ende des 18. oder Anfang des 19. Jahrhunderts entstanden sind. Die Münchener Partitur (D-Mbs, Mus.ms. 822) stammt aus dem Besitz des Tübinger Juristen und Musikhistorikers Anton Friedrich Justus Thibault (1772-1840), bei dem Schreiber könnte es sich um einen Kopisten aus der Werkstatt des Mainzer Musikalienhändlers Georg Carl Zulehner handeln, von dem Thibault zahlreiche Abschriften erwarb. ${ }^{1}$ Das weniger bekannte Hamburger Exemplar, das vermutlich etwas später entstand, stammt aus dem Besitz Friedrich Chrysanders. ${ }^{2}$ Beide Quellen stehen in einem direkten Zusammenhang zueinander, da sie die gleichen schweren Textierungsfehler aufweisen, die Schreiber scheinen über keine oder nur unzureichende Italienischkenntnisse verfügt zu haben; insgesamt ist aber die Münchener Abschrift bei den meisten Lesarten die zuverlässigere Quelle.

Erstmals beschäftigte sich Carl Mennicke mit der Musik des Oratoriums, dabei unterzog er die instrumentale Einleitung einer kritischen Würdigung. Obwohl es sich um eine geradezu typische Wiener Kirchensinfonie aus langsamer Einleitung und fugiertem schnellen Teil handelt, wie wir sie auch in den Oratorien Caldaras, Fux' und Reutters finden, kritisiert Mennicke den angeblich zu weltlichen Tonfall des fugierten Abschnittes, desssen sprunghafte Melodik er als unangemessen für das ernste Thema des Werkes empfindet. Schon Mennicke bezweifelte allerdings die Authentizität der Partitur, ohne diese jedoch eingehender zu diskutieren. ${ }^{3}$

Łucjan Kamieński betonte in seiner Monographie zu Hasses Oratorien von 1912 noch einmal die erheblichen Zweifel an der Echtheit des Werkes. ${ }^{4}$ Er urteilte recht negativ über die Partitur und schloss auf Grund stilistischer Kriterien und der seiner Ansicht nach geringen musikalischen Qualität eine Autorschaft Hasses aus. Dabei hob er die fehlerhafte Textdeklamation (die allerdings teilweise Schuld des Kopisten war), die untypische Behandlung der Singstimmen, die für ein Passionsoratorium sehr unspezifische Affektsprache und den im Vergleich zu Hasses bekannten Oratorien sehr weltlichen Tonfall hervor.

Eine genauere musikalische Einordnung des rätselhaften Werkes gelang erst 1976 mit Reinhard Strohms Dissertation zu italienischen Opernarien des frühen 18. Jahrhunderts. ${ }^{5}$ Er konnte nachweisen, dass das Stück ein Pasticcio ist, ihm gelang es dabei, einen großen Teil der in der Partitur enthaltenen Arien zu identifizieren. Sie stammen

1 Nach älterer bibliothakarischer Überlieferung soll Zulehner auch der Schreiber der Partitur sein, dies ließ sich jedoch durch Schriftvergleiche nicht bestätigen.

2 D-Hs, ND VI 2561, Handschrift aus dem Besitz von Friedrich Chrysander. Vgl. die Beschreibung der Hamburger Quelle von Hans-Jörg Drauschke, Hamburg 2003, online verfügbar unter https://www.sub.unihamburg.de/fileadmin/redaktion/HAC_2016/Hasse/Oratorien/nd_vi_2461.pdf (abgerufen am 23. 2. 2018).

3 MENNICKE, Carl. Hasse und die Brüder Graun als Symphoniker: nebst Biographien und thematischen Katalogen. Leipzig 1906, S. 189-191. Dort auch eine Edition des Beginns der Introduzione.

4 KAMIEŃSKI, Łucjan. Die Oratorien von Johann Adolf Hasse. Leipzig 1912, S. 216-217.

5 STROHM, Reinhard. Italienische Opernarien des frühen Settecento (1720-1730). vol. II: Notenbeispiele und Verzeichnisse. Köln 1976, S. 277-278. 
demnach aus Opern, die zwischen 1728 und 1733 in Italien uraufgeführt wurden, überwiegend in Bologna, Neapel und Venedig. Da die Münchener Quelle auf Grund ihres späten Entstehungsdatums jedoch keine genauere musikhistorische und geographische Einordnung erlaubte, konnte er keine weiteren sicheren Angaben zu Herkunft und Entstehungsanlass des Pasticcios ermitteln. Er äußerte lediglich die Vermutung, das Werk könne aus der Umgebung des Münchener Hofes stammen, wofür es aber abgesehen von dem Aufbewahrungsort der Partitur keinen plausiblen Hinweis gibt, zumal diese Form von Oratorien für München äußerst untypisch gewesen wäre. Strohms Identifizierungen erklärten die Zuschreibung der Partitur an Hasse, da vier der enthaltenen Arien aus Opern Hasses stammen, drei davon aus seinem 1733 mit großem Erfolg in Bologna aufgeführten Siroe, eine Tatsache, die Kamieński erstaunlicherweise übersah. ${ }^{6}$ Die weiteren von Strohm identifizierten Komponisten sind Leonardo Leo, Francesco Feo und Giuseppe Porta.

Alle weiteren Erwähnungen der Partitur Hasses bringen keine Erkenntnisse, die über die von Strohm hinaus ermittelten Tatsachen hinausgehen. Etwas ausführlicher widmete sich noch einmal Michael Koch in einer neueren Studie zum Oratorienschaffen Hasses dem Werk. ${ }^{7}$ Da er Strohms Arbeit nicht kannte, ging er allerdings von völlig falschen Prämissen aus, zudem unterliefen ihm einige schwerwiegende Fehler: So erkannte er nicht die späte Entstehung der Quelle; von späterer Hand eingetragenen Korrekturen und Ergänzungen deutete er versehentlich als autographe Einträge Hasses. Zudem las er die Tenorschlüssel in der Partitur (für die Partien des Giovanni und des Cristo) versehentlich als Altschlüssel, was ihn zu der falschen Behauptung verleitete, das Oratorium sei ausschließlich für Frauenstimmen besetzt und somit vermutlich für eines der venezianischen Mädchen-Konservatorien bestimmt gewesen. ${ }^{8} \mathrm{Da}$ an den venezianischen Ospedali ausschließlich Oratorien in lateinischer Sprache aufgeführt wurden, kann unser italienisches Passionsoratorium unmöglich diesem Kontext zugeordnet werden.

Seit Strohms bahnbrechender Studie von 1976 haben sich die Recherchemöglichkeiten für die Erforschung des Opernrepertoires des 18. Jahrhunderts erheblich verbessert. Digitalisierte, über Datenbanken ermittelbare Libretti und Musikhandschriften sowie die Incipitrecherche des RISM-Opac führen inzwischen innerhalb kurzer Zeit zu Ergebnissen, für die vor einigen Jahren noch langwierige Forschungen nötig waren. So konnte ein weiterer Satz aus dem Oratorium durch Inzipitvergleiche als Opernarie identifiziert werden: „Pensa tu che sei clemente“ ist die Kontrafaktur der Arie „Vi sarà stella clemente“ aus Giovanni Battista Pescettis Oper I tre difensori della Patria (Venedig 1729), eine Arie, die

6 Strohm schrieb sogar fünf Arien Hasse zu. In einem Fall unterlief ihm dabei eine Verwechslung. „Che in me si freni il pianto“ stammt nicht aus Hasses Demetrio (Venedig 1732), sondern aus Leonardo Leos im gleichen Jahr in Neapel entstandener Vertonung desselben Librettos. Vgl. die Partitur der Oper in I-Mc, Fondo Noseda F 92.

7 KOCH, Michael. Die Oratorien Johann Adolf Hasses. Überlieferung und Struktur (= Musikwissenschaftliche Studien 14). Pfaffenweiler 1989, S. 19-23.

8 Tatsächlich sehen die C-4-Schlüssel des Kopisten wie C-3-Schlüssel aus, doch hätte sich dieses Missverständnis bei oberflächlicher Betrachtung der Melodik der Singstimmen eigentlich auflösen müssen. 
auch in den in Darmstadt und Regensburg überlieferten Partituren des aus der VivaldiForschung bekannten Opernpasticcios Argippo enthalten ist. ${ }^{9}$

Letztendlich gelang es durch die Entdeckung eines konkordanten Librettos, die Herkunft des Pasticcios aufzudecken. Es handelt sich um ein einen Prager Textdruck von 1744 mit dem Titel La Vittima d'amore ossia la morte di Cristo, der weitgehend mit dem Gesangstext der Münchener Partitur übereinstimmt. ${ }^{10}$ Das Oratorium erklang am Vormittag des Karfreitags in der Kirche der Kreuzherren zum Roten Stern. Da der Text des Oratoriums Paraphrasen von übernommenen Opernarien enthält, deren Wortlaut zum Teil eng an der Vorlage gehalten ist, darunter bekannte Metastasio-Texte, konnte es sich nicht um eine andere Vertonung des gleichen Textes handeln, die Partitur enthält also mit großer Sicherheit die Musik, die 1744 in Prag erklang. Die dichterische Vorlage für das Pasticcio - dessen archaischen Charakter bereits Michael Koch bemerkte - ist ein altes Libretto von Francesco Torti, das 1690 von Antonio Giannettini in Modena vertont wurde. Eine überarbeitete Fassung mit neu gedichtetem Schlusschor wurde 1695 aufgeführt, wie das erhaltene Libretto zu dieser Aufführung bezeugt, ${ }^{11}$ und diese Fassung gelangte schließlich an den Wiener Hof, wo das Werk 1704 erklang. ${ }^{12}$ Größter Eingriff gegenüber der Vorlage Tortis ist die Einführung einer vierten Rolle, eines Engels, der jeden der beiden Teile mit einer Art Prolog eröffnet und an Stelle des Chors eine Arie am Abschluss des ersten Teils singt. Im Prager Libretto sind die Stimmlagen der vier Solisten genannt: Angelo, Maria Vergine, S. Giovanni und Cristo sind demnach jeweils mit Sopran, Alt, Tenor und Bass besetzt. Anders in den erhaltenen Partituren: dort sind die Rollen mit 2 Sopranen und 2 Tenören besetzt. Verwunderlich erscheint auch ein weiterer Befund: Im Libretto ist ein einzelner Komponist als Autor der Musik genannt, was dem nachweisbaren Pasticcio-Charakter des Werkes widerspricht. Es handelt sich dabei um Josef Umstatt, der damals in Brünn als Kapellmeister des Grafen Leopold von Dietrichstein (1703-1773) wirkte. ${ }^{13}$ Das Oratorium muss in Deutschland auch unter Um-

9 Siehe hierzu STROHM, Reinhard. Argippo in „Germania“. Studi vivaldiani 8, 2008, pp. 111-126. Nachweis der Arie auf S. 125. Zur Darmstädter Quelle, die bei Strohm noch nicht diskutiert wurde, siehe PEGAH, Rashid-Sascha. Ein Argippo-Pasticcio. Studi vivaldiani 11, 2011, pp. 63-76. In den Prager und Wiener ArgippoFassungen taucht die Arie nicht auf.

10 La Vittima d'Amore ó Sia la Morte di Giesù Christo Salvatore Nostro Oratorio per Musica, Prag 1744, Exemplar in der Wissenschaftlichen Bibliothek Olmütz, Signatur 33.498.

11 La Vittima d'Amore o sia La morte di Cristo Oratorio del Sig. Dott. Francesco Torti Musica del Sig. Antonio Gianettini Maestro di Capella di S.A.S., Modena, Bartolomeo Soliani 1695. Exemplar in I-MOe, 70.I.23 (5)

12 Signatur der Wiener Partitur von Giannettinis Oratorium: A-Wn, Mus.ms. 17.684. Zur Datierung der Wiener Aufführung vgl. WEILEN, Alexander von. Zur Wiener Theatergeschichte. Die vom Jahre 1629 bis zum Jahre 1740 am Wiener Hofe zur Aufführung gelangten Werke theatralischen Charakters und Oratorien, Wien 1901, S. 64. Ein Vergleich mit der Wiener Partitur ergab, dass das Pasticcio-Oratorium weist keinerlei musikalische Übereinstimmungen mit Giannettinis Werk aufweist.

13 Zu Umstatts Biographie und seinem Wirken in Mähren vgl. KAČIC, Ladislav. Joseph Umstatt (1711-1762) zwischen Barock und Klassik: Bemerkungen zur Stilentwicklung eines mitteleuropäischen Komponisten. In Zur Geschichte und Aufführungspraxis der Musik des 16.-18. Jahrhunderts in Mittel- und Osteuropa. G. Gruber (Ed.). Bratislava, 2013, pp. 201-215. 
statts Namen bekannt gewesen sein; die Notenbibliothek der Thomasschule in Leipzig besaß eine (heute verschollene) Partitur des Werkes. ${ }^{14}$

In Prag wurde das Oratorium allerdings nicht zum ersten Mal gespielt: Die Uraufführung fand vermutlich 1741 in Brünn statt, also dem Jahr, in dem Umstatt in die Dienste des Grafen von Dietrichstein trat, auch für diese Aufführung ist ein Libretto erhalten. ${ }^{15}$ Es kann davon ausgegangen werden, dass der als Komponist genannte „Giuseppe Umstatt" der Arrangeur des Pasticcios war, sicherlich komponierte er auch die Rezitative, die instrumentale „Introduzione“ sowie den dreistimmigen "Coro degli Angeli“ am Ende des Werkes.

Jana Spáčilová wies erstmals auf den aus dem Libretto herauslesbaren Pasticcio-Charakter des Oratoriums hin, als sie das Brünner Libretto in dem Kontext anderer italienischer Oratorien, die um 1730 in Brünn aufgeführt wurden, untersuchte; dabei fielen ihr vor allem die Metastasio-Paraphrasen auf, insbesondere die bekannte Siroe-Arie „Gelido in ogni vena", die hier von der Figur des Giovanni gesungen wird. Vor allem konnte sie eine Arie als Entlehnung aus dem 1724 in Wien entstandenen Passionsoratorium Morte e sepoltura di Cristo von Antonio Caldara identifizieren. Caldaras Oratorium wurde bereits 1730 in Brünn nachgespielt. Eine in Berlin überlieferte Abschrift des Oratoriums, die von einem Salzburger Schreiber stammt, kann eindeutig der Brünner Aufführung zugeordnet werden, wie Spáčilová in einem älteren Aufsatz nachwies. ${ }^{16}$ In dieser Partitur wurde unter den Originaltext der Arie der Maria Giacobbe „Lasciami eterno amante“ nachträglich ein neuer Parodietext unterlegt, es handelt sich um jenen Arientext „Schernito e disprezzato" aus dem Libretto zu dem Pasticcio-Oratorium. ${ }^{17}$ Auch die Münchener Pasticcio-Partitur enthält, wie zu erwarten war, die Arie Caldaras mit dem neuen Parodietext.

Rechnet man die Funde Strohms, die von Spáčilová entdeckte Caldara-Parodie und die über die RISM-Recherche ermittelte Pescetti-Arie zusammen, bleiben nur noch drei Arien übrig, für die keine Vorlage ermittelt werden konnte; es könnte sich bei ihnen um Originalkompositionen Umstatts handeln. Danach ergibt sich die folgende Struktur für das Werk:

14 Vgl. den Eintrag zu Umstatt bei Eitner, Joseph. Biographisch - Bibliographisches Quellen-Lexikon der Musiker und Musikgelehrten der christlichen Zeitrechnung bis zur Mitte des 19. Jahrhunderts. Band 10, Leipzig 1904, S. 10: „La Vittima d'amori [sic!] osia La morte de Christe [sic!]. Ms. P., italienisch und deutsch.“

15 SPÁČILOVÁ, Jana. Unbekannte Brünner Oratorien neapolitanischer Komponisten vor 1740. Musicologica Brunensia 49, 2014, iss. 1, pp. 137-161, besonders S. 142-144.

16 SPÁČILOVÁ, Jana. Die Rezeption der italiensichen Oper am Hofe des Olmützer Bischofs Schrattenbach. In The Eighteenth-Century Italian Opera Seria. Metamorphoses of the Opera in the Imperial Age (=Colloquium Musicologicum Brunense 42: 2007). Eds. Petr Macek and Jana Perutková. Praha 2013, pp. 75-88. Zur Brünner Provenienz der Berliner Partitur D-B, Mus.ms. 2720 vgl. S. 81-86.

17 SPÁČILOVÁ, Jana. Unbekannte Brünner Oratorien neapolitanischer Komponisten vor 1740, op. cit., S. 144. In der Münchener Abschrift des Caldara-Oratoriums (D-Mbs Mus.ms. 715), die bezeichnenderweise ebenfalls aus der Sammlung Thibaut stammt, findet sich unter der Arie die gleiche Doppeltextierung. Die Abschrift wurde also nach der Berliner Vorlage vorgenommen. 
Das Johann Adolf Hasse zugeschriebene Passions-Oratorium La morte di Cristo ...

\begin{tabular}{|c|c|c|}
\hline La Vittima d'amore / La morte di Cristo & Komponist und Vorlage & Originaltext \\
\hline \multicolumn{3}{|l|}{ Introduzione } \\
\hline \multicolumn{3}{|l|}{ Parte prima: } \\
\hline \multicolumn{3}{|l|}{ Recit. Troppo Signor troppo orgoglioso } \\
\hline Aria (Angelo) Dalle fiamme e dalle straggi & $\begin{array}{l}\text { Leonardo Leo: Argeno } \\
\text { (Venedig 1728) }\end{array}$ & $\begin{array}{l}\text { Nelle fiamme e nelle } \\
\text { stragi }\end{array}$ \\
\hline \multicolumn{3}{|l|}{ Recitativo. Già decreto è del padre } \\
\hline Aria (Maria) Se il tuo penar mi rendi & $\begin{array}{l}\text { Johann Adolf Hasse: Siroe (Bo- } \\
\text { logna 1733) }\end{array}$ & Se l'amor tuo mi rendi \\
\hline \multicolumn{3}{|l|}{ Recit. Egli è ver ma sei donna } \\
\hline \multicolumn{3}{|l|}{ Aria (Giovanni). Fa pur che resti spento } \\
\hline \multicolumn{3}{|l|}{ Recit. È ver che uom tu sei } \\
\hline Aria (Cristo) Reo si fa d'un alto sdegno & Leonardo Leo: Argeno & Cheto sta nella foresta \\
\hline \multicolumn{3}{|l|}{ Recit. Gran cifre mi snodasti } \\
\hline Aria (Giovanni) Pensa tu che sei clemente & $\begin{array}{l}\text { Giovanni Battista Pescetti: } \\
\text { I tre difensori della patria (Ve- } \\
\text { nedig 1729) }\end{array}$ & Vi sarà stella clemente \\
\hline \multicolumn{3}{|l|}{ Recit. Chi pena per amore } \\
\hline Aria (Maria) Schernito e disprezzato & $\begin{array}{l}\text { Antonio Caldara: Morte e se- } \\
\text { poltura di Cristo (Wien 1724) }\end{array}$ & Lasciami eterno amante \\
\hline \multicolumn{3}{|l|}{ Recit. Se il pianto ed il dolore } \\
\hline Aria (Angelo) Già vassi il diletto & $\begin{array}{l}\text { Giovanni Porta: Farnace (Bolo- } \\
\text { gna 1731) }\end{array}$ & Mi parla nel seno \\
\hline \multicolumn{3}{|l|}{ Parte seconda: } \\
\hline \multicolumn{3}{|l|}{ Recit. Non basterà lo sdegno } \\
\hline Aria (Angelo) Quale ai venti esposta nave & $\begin{array}{l}\begin{array}{l}\text { Francesco } \\
\text { (Rom 1728) }\end{array} \\
\text { (Reo: Ipermestra } \\
\end{array}$ & A due venti esposta nave \\
\hline \multicolumn{3}{|l|}{ Recitativo accomp. Mirate o cieli o stelle } \\
\hline \multicolumn{3}{|l|}{ Aria (Cristo) Voi del ciel lumi che siete } \\
\hline \multicolumn{3}{|l|}{ Recit. Se piangeran le stelle } \\
\hline Aria (Maria) Quella che a te la vita & Johann Adolf Hasse: Siroe & Ebbi da te la vita \\
\hline \multicolumn{3}{|l|}{ Recit. Ei pena e soffre } \\
\hline Aria (Giovanni) Gelido in ogni vena & Johann Adolf Hasse: Siroe & Gelido in ogni vena \\
\hline \multicolumn{3}{|l|}{ Recit. A questi colpi o figlio } \\
\hline Aria (Maria) Che in me si freni il pianto & Leo: Demetrio (Neapel 1732) & Di quell'ingiusto sdegno \\
\hline \multicolumn{3}{|l|}{ Recit. Donna troppo t'affanni } \\
\hline Aria (Giovanni) Impallidisce il sole & $\begin{array}{l}\text { Johann Adolf Hasse: Issipile } \\
\text { (Neapel 1732) }\end{array}$ & Impallidisce in campo \\
\hline \multicolumn{3}{|l|}{ Recit. accomp. Dell'opra mia si grande } \\
\hline \multicolumn{3}{|l|}{ Aria (Cristo) S'armin pure a mille } \\
\hline \multicolumn{3}{|l|}{ Recit. accomp. Agonizzante ancora } \\
\hline Coro d'angioli. Così muore e lo stupor & & \\
\hline
\end{tabular}

Ein instruktives Beispiel für die Problematik der Pasticcio-Technik ist die Arie „Impallidisce il sole“ aus dem 2. Teil, die aus Hasses 1732 in Neapel uraufgeführter Issipile stammt, im Original eine Bravourarie für die Primadonna Faustina Bordoni. Im Oratorium wurde die Arie für die Partie des S. Giovanni, eine der beiden Tenorrollen des Stückes, eine Oktave tiefer transponiert, ansonsten blieb die Musik unverändert. Anbei ein Vergleich 
zwischen dem Originaltext Metastasios und der Paraphrase aus dem Passions-Libretto:

Metastasio, Issipile

Impallidisce in campo

Anche il guerrier feroce

A quella prima voce

Che all'armi lo destò.

D'ardir non è difetto

Quel resto di timore

Che nel fuggir dal petto

Sul volto si fermò.
La vittima d'amore (Brno 1741)

Impallidisce il sole

Spesso tra l'auree stelle

E per dar fasto a quelle

Egli s'asconde in mar.

Così l'autor del giorno

Depone i pregi suoi

E per dar vita a noi

Estinto vuol restar.

In der heroischen Arie der Titelheldin aus dem ersten Akt der Oper argumentiert Issipile, von ihrer Widersacherin Eurinome der Feigheit bezichtigt, dass äußerliche Anzeichen von Furcht keinen Mangel an Entschlossenheit bedeuten. In Hasses Vertonung ahmen die triumphalen Fanfaren der Hörner jene im Text erwähnte „erste Stimme“, die den Krieger zur Schlacht ruft, nach; der kriegerische Charakter spiegelt sich in den brillanten Koloraturketten der Singstimme wieder. Von dem im Gedicht thematisierten Erblassen des Kriegers, eine instinktive Reaktion auf den bevorstehenden Kampf, erzählt Hasses Musik hier nichts. ${ }^{18}$

Der neue Arientext des Oratoriums behält von Metastasios Vorlage nur das erste Wort „Impallidisce“ bei, ansonsten gibt es keine textlichen Übereinstimmungen. Es handelt sich um ein eschatologisches Gleichnis, in dem der sich für die Menschheit opfernde Christus mit der Sonne verglichen wird, die sich am Abend ins Meer zurückzieht, um die kleineren Sterne des Himmels - ein Abbild der sündigen Menschen - leuchten zu lassen. Das neue Gedicht hat also einen völlig anderen Affektgehalt, und würde eigentlich eine dunklere, getragene Stimmung erwarten lassen; Hasses auftrumpfende Bravourarie wirkt angesichts der dramatischen Situation - Johannes und Maria, die am Kreuz zu Zeugen des Todeskampfes Christi werden - nicht wirklich angemessen. Am ehesten ließe sich die musikalische Einkleidung noch mit dem „fasto“ der Sterne erklären, die aber gar nicht Hauptgegenstand des Gedichtes sind. Rechtfertigen lässt sich der kämpferische Charakter der Arie theologisch allerdings durch die Deutung der Passion als Sieg des „Helden aus Juda“, wie es vor allem in der Interpretation des Johannes-Evangeliums immer wieder betont wird, gerade weil die Arie ja hier von dem Jünger gesungen wird, der nach traditioneller Auffassung Verfasser des Evangeliums war.

Stilistisch stark abweichend von den paraphrasierten neapolitanischen Opernarien sind die drei bisher nicht identifizierten Arien sowie die einleitende Sinfonia und der für drei hohe Stimmen (2 Soprane und Alt) und Streicher gesetzte Schlusschor, der einzige madrigalische Text, der aus der Originaldichtung Fortis beibehalten wurde. Die bereits von Mennicke besprochene Sinfonia folgt dem Modell der Oratoriensinfonien Caldaras,

18 Nachzuhören ist die Arie in der Interpretation von Vivica Genaux auf der CD „Rival Queens“ unter der Leitung von Andrès Gabetta, erschienen 2014 bei Sony Classics. 
mit einer kurzen langsamen Einleitung, die vom für den Kirchenstil typischen Durezze e ligature - Stil geprägt ist, und eine ausgedehnte Vivace-Fuge im 3/8-Takt. Bisher konnte keine Vorlage für das Stück ermittelt werden, eine Autorschaft des Fux-Schülers Umstatt, der einen hervorragenden Ruf als Kontrapunktiker genoss, bleibt wahrscheinlich. Auch der kurze, aber harmonisch dichte Schlusschor für drei hohe Stimmen ist sicherlich eine Originalkomposition für das Oratorium. (Sinfonia und Anfang des Chors als Notenanhang 1 und 3 am Ende des Artikels)

Von den drei unbekannten Arien stellt die erste, Giovannis „Fa pur che resti spento“, die meisten Fragen. Es handelt sich um eine schlichte Menuett-Arie (G-Dur, 3/8-Takt), die mit Streichern (vermutlich durch Flöten verdoppelt) und 2 obligaten Bass-Instrumenten, sicherlich Fagotten, besetzt ist. Zunächst fällt die Notation der Singsstimme im Diskantschlüssel auf; da Giovanni eine Tenor-Partie ist, muss sie eine Oktave tiefer gelesen werden. Des Weiteren bricht in den beiden erhaltenen Partituren die Textierung der Singstimme am Ende des A-Teils ab. Bei genauerer Betrachtung des Gesangstextes fällt dann auf, dass eine metrisch korrekte Unterlegung unter die vorhandene Singstimme sehr problematisch ist, wie die ersten Takte der Singstimme zeigen:

"Fa pur che resti spento / In me quell'uom, quell'empio / Che incauto volle errar"

Die Melodie scheint tatsächlich für einen Vers aus drei Ottonari komponiert zu sein, wie auch die Balkung der Singstimme des ersten Taktes zeigt; für die Unterlegung der Settenari des vorliegenden Gedichtes müssen Kompromisse wie der Oktavsprung auf einer Silbe im 5. Takt eingegangen werden. Deswegen erscheint es unwahrscheinlich, dass hier eine Originalvertonung vorliegt.

Anders sieht es bei den beiden Christus-Arien im zweiten Teil des Oratoriums aus. Beide werden durch ausdrucksvolle Accompagnato-Rezitative eingeleitet. Stilistisch setzen sich diese Stücke stark von den neapolitanischen Pasticcio-Arien ab, sie entsprechen eher dem hochbarocken Stil Caldaras. Da in beiden Fällen die Textunterlegung ohne „Verrenkungen“ möglich ist und auch der Affekt des Gesangstextes gut getroffen ist, könnten diese Arien tatsächlich von Umstatt für das Oratorium neu komponiert worden sein. Außergewöhnlich ist vor allem die e-moll-Arie „Lumi voi che siete“, die von einem reich modulierenden Rezitativ eingeleitet wird. ${ }^{19}$ Die Arie steht durchgängig ist in einem realen fünfstimmigen Satz, in scharfem Kontrast zu den galanten, durchsichtig gesetzten Opernarien der „Neapolitaner“. Der Affekt des Schmerzes wird einerseits durch die sequenzierende Basslinie, die einen fallenden Tetrachord umfasst, ausgedrückt, andererseits durch Dissonanzen und „schwierige“ querständige Tonverbindungen in der Singstimme, gipfelnd in den gequälten Melismen auf dem Wort „dolor“ im B-Teil der Arie.

In seiner Synthese aus moderner neapolitanischer Opernmusik und traditionellen Elementen des Wiener Barockoratoriums ist La vittima d'amore somit ein typisches Beispiel für den stilistischen Synkretismus, der charakterisch ist für die Brünner Oratorienpflege der dreißiger und vierziger Jahre des 18. Jahrhunderts. Interessant ist hierbei die Beobachtung, dass ein relativ junger Komponist wie Joseph Umstatt dabei als Vertreter der älteren Tradition in Erscheinung tritt.

19 Vergleiche Notenanhang 2 am Ende des Artikels. 


\section{Anhang 1}

\section{La morte di Cristo}

Introduzione
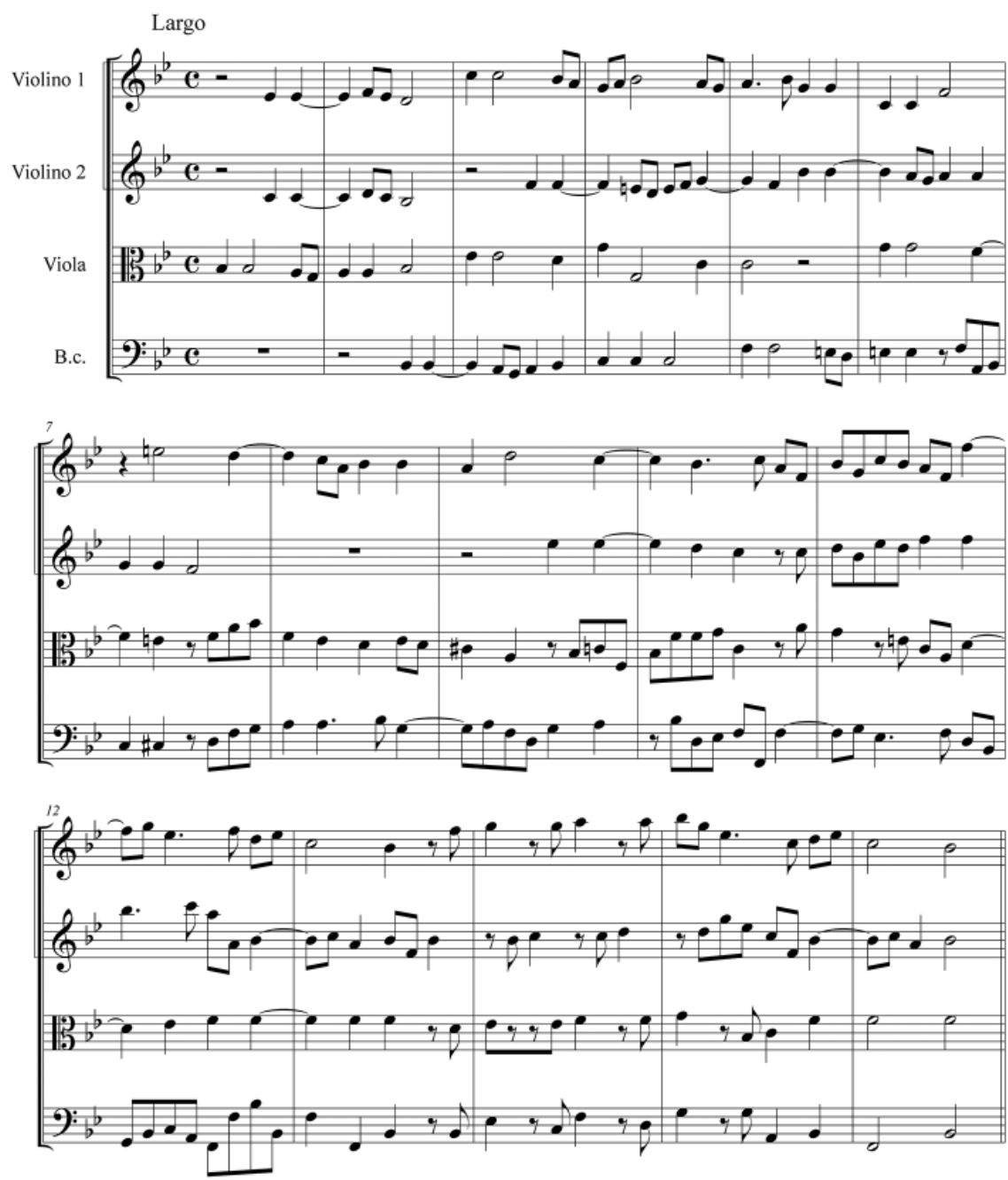
Steffen Voss

Das Johann Adolf Hasse zugeschriebene Passions-Oratorium La morte di Cristo ...
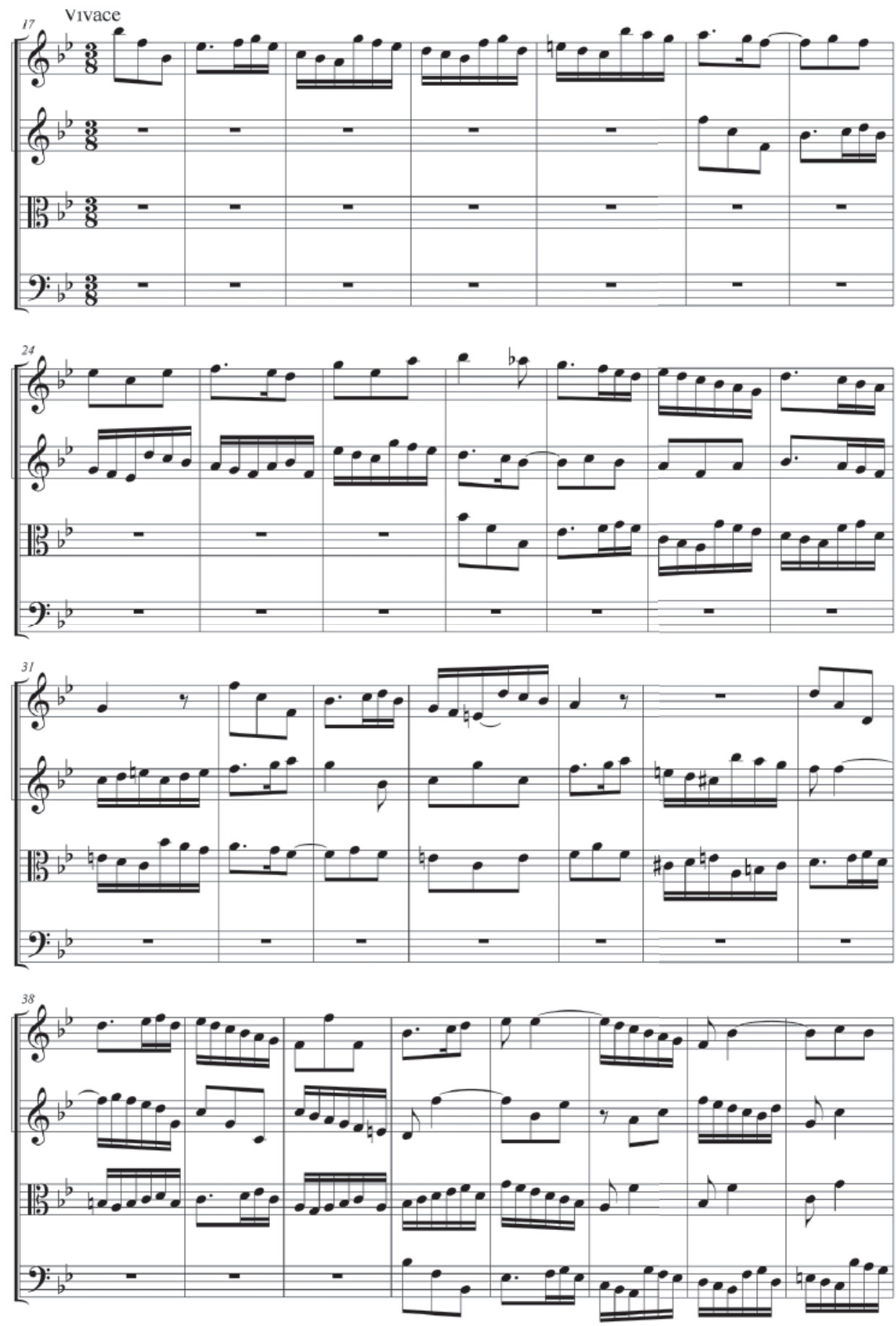

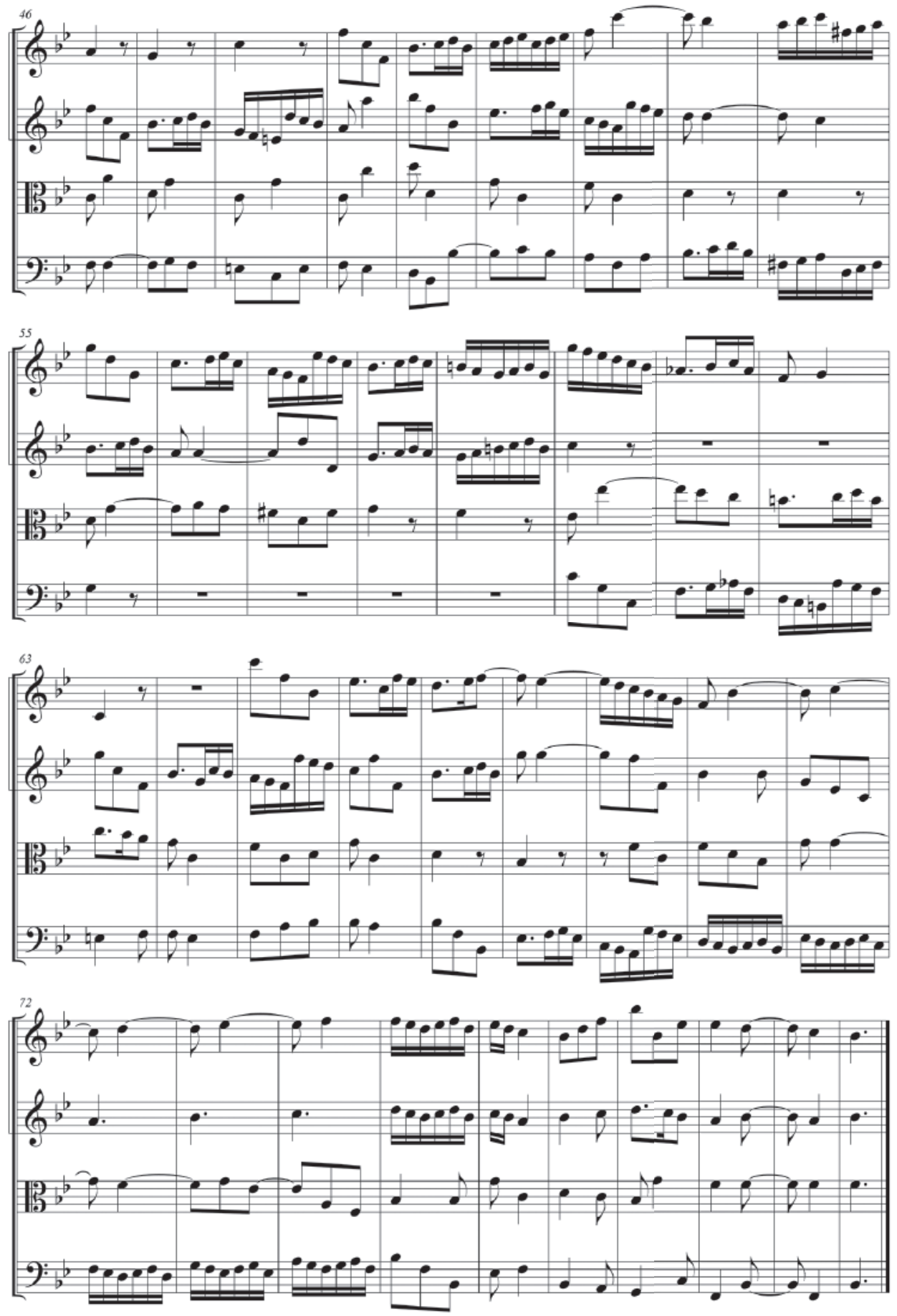


\section{Anhang 2}

Recitativo accompagnato
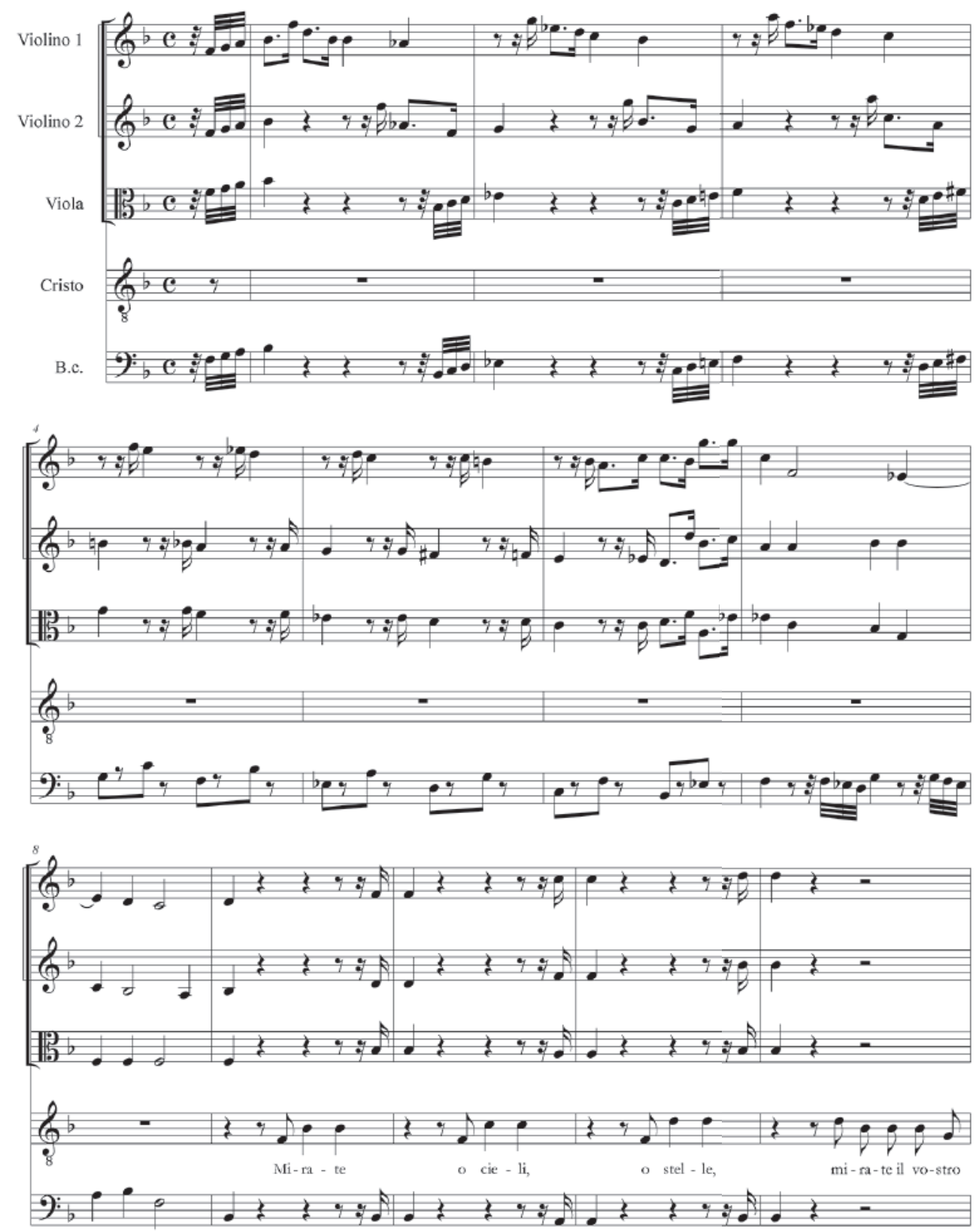
Recitativo
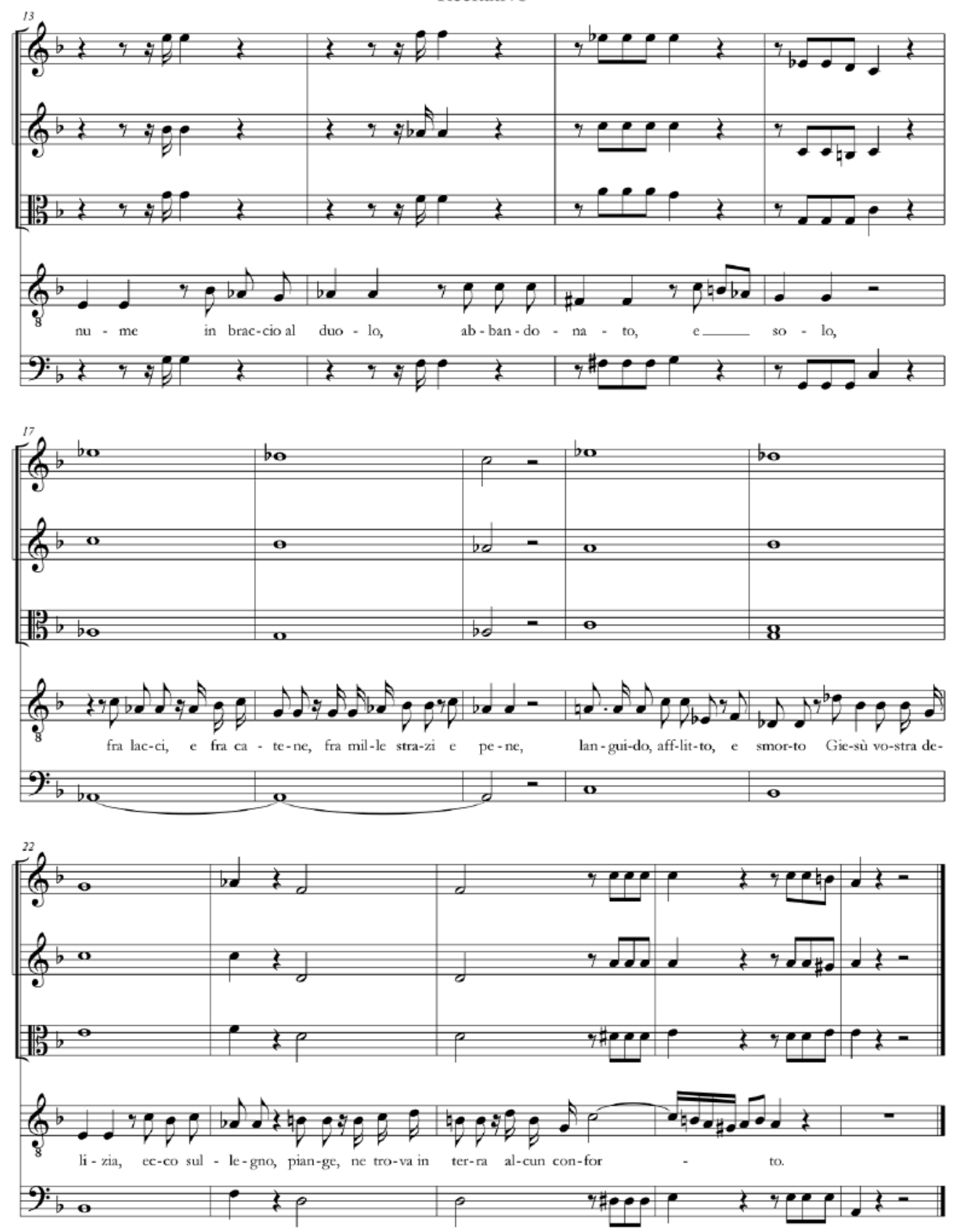
Affetuoso e dolce

\section{Aria}
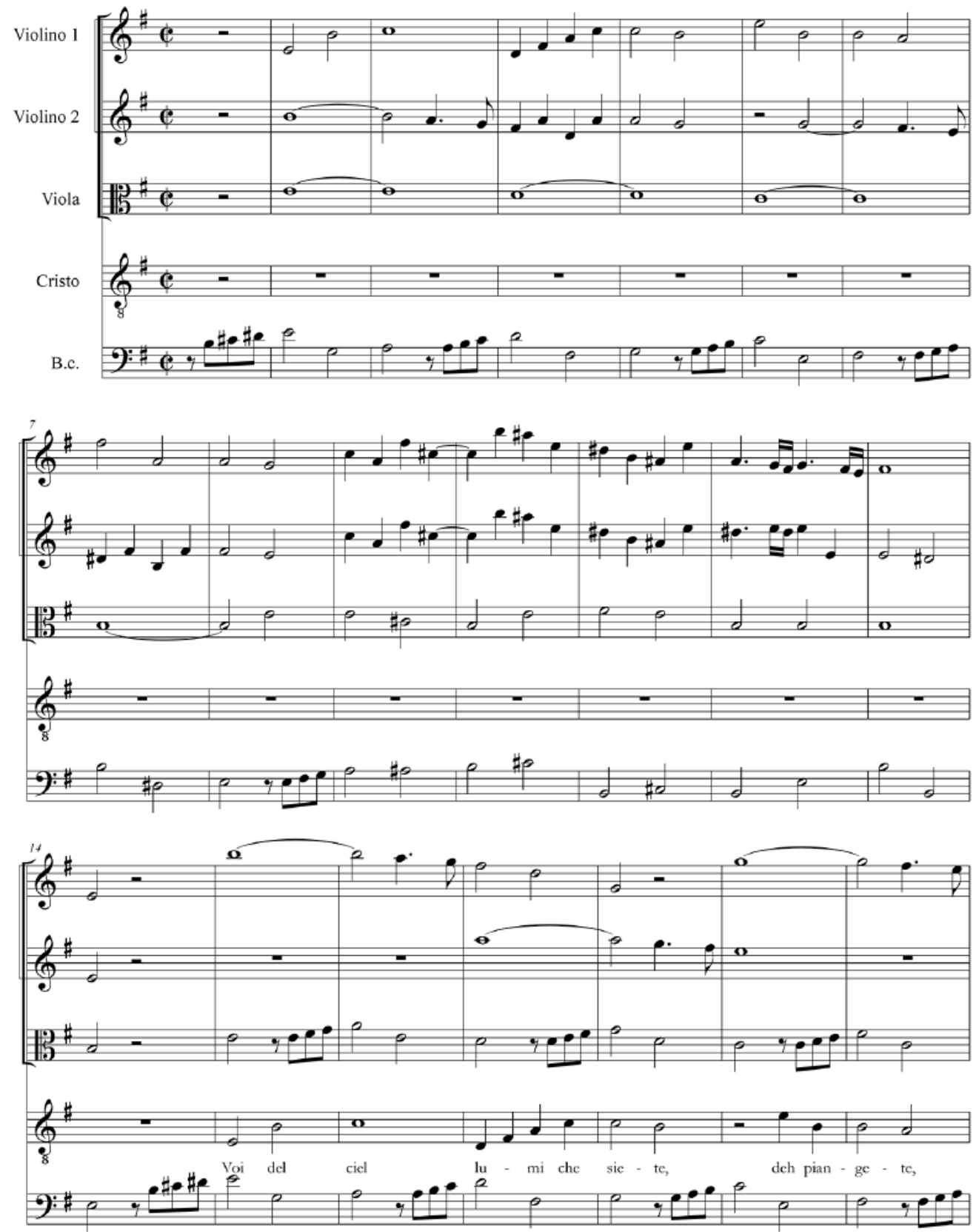

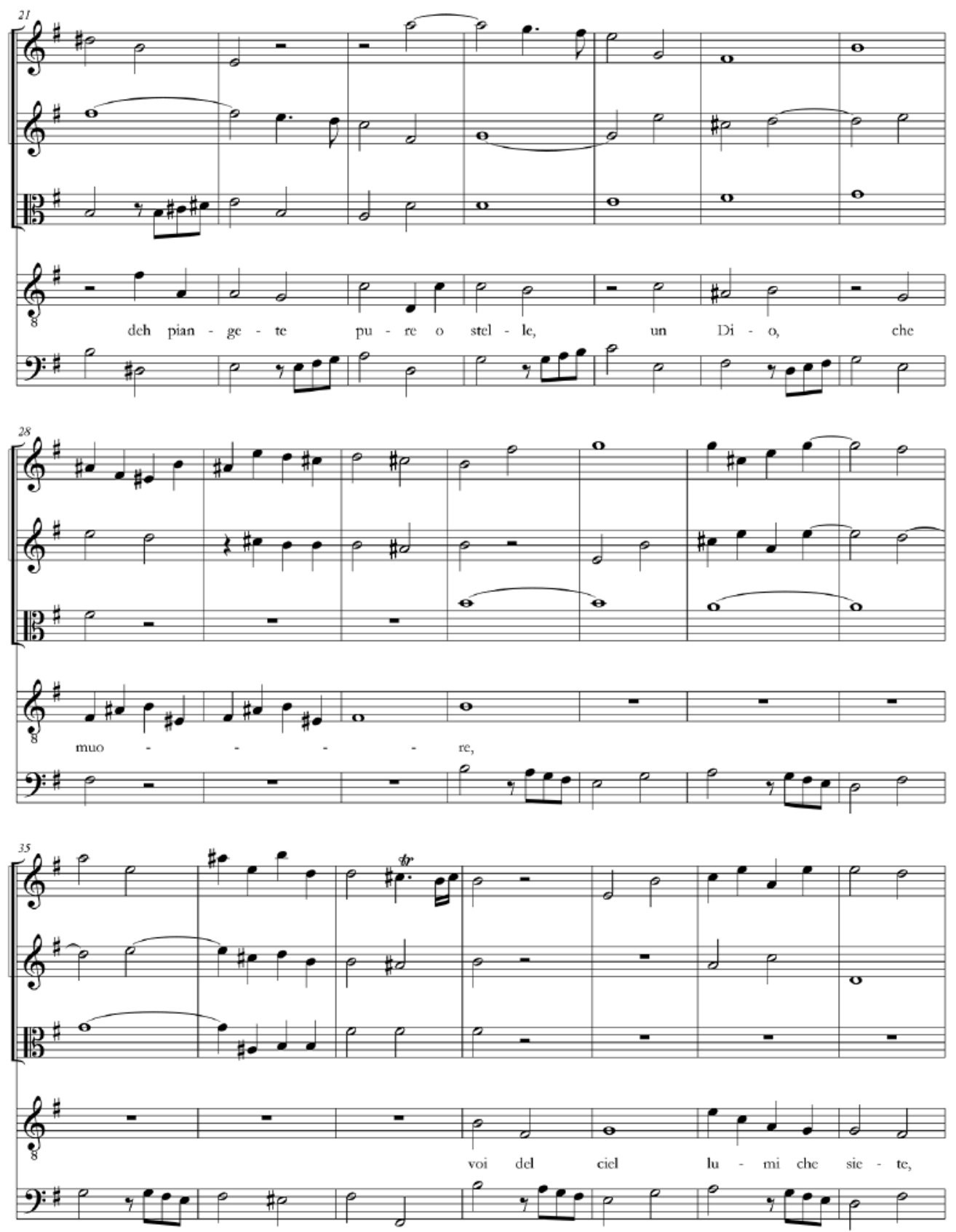
Steffen Voss

Das Johann Adolf Hasse zugeschriebene Passions-Oratorium La morte di Cristo ...
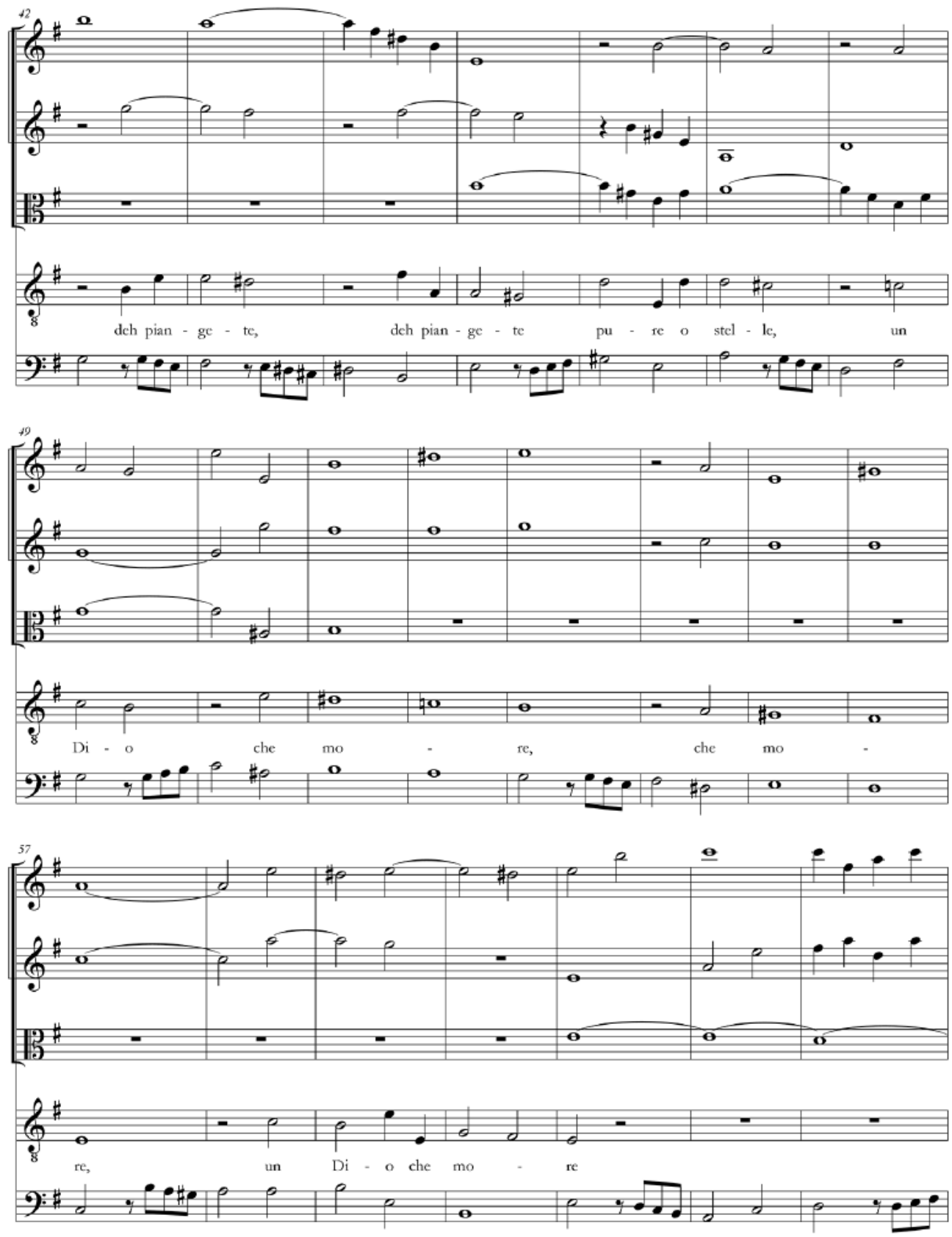

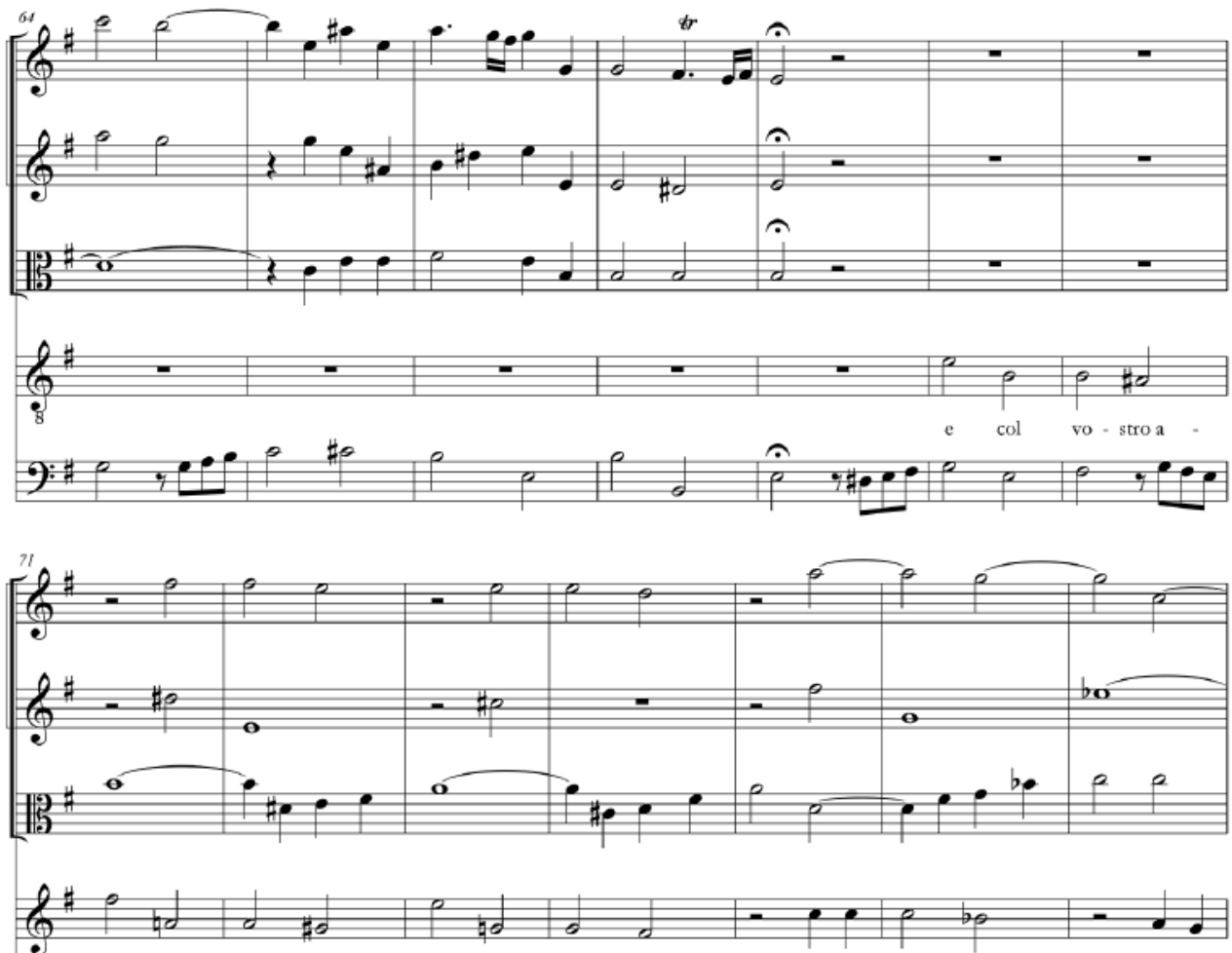

ma - ro pian - to rot frat - tan - to ris-pon - de - te, ris-pon-

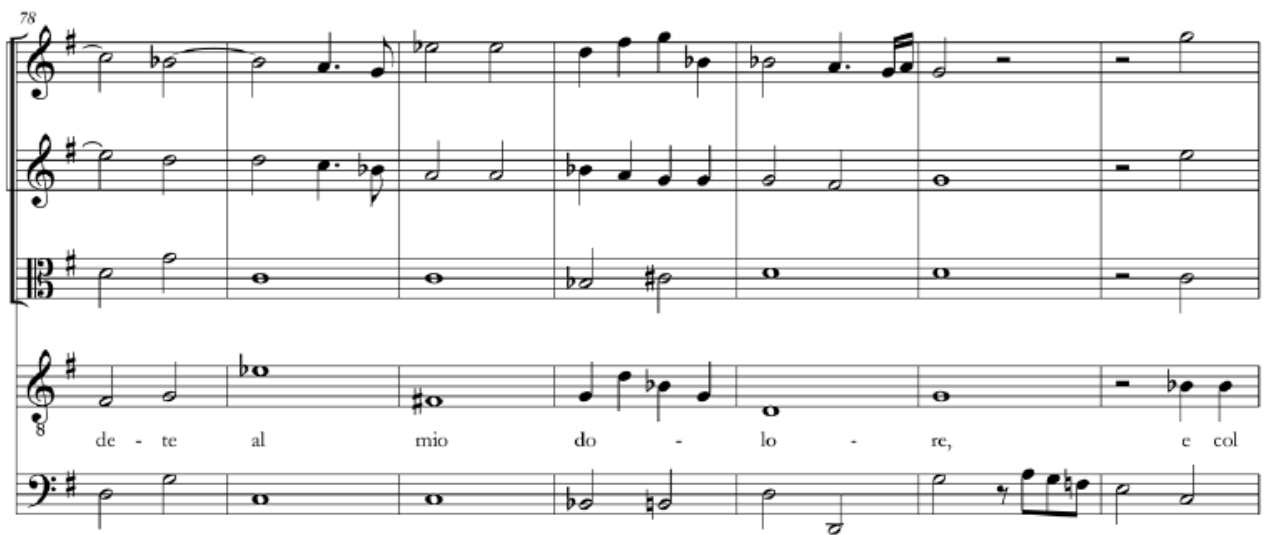



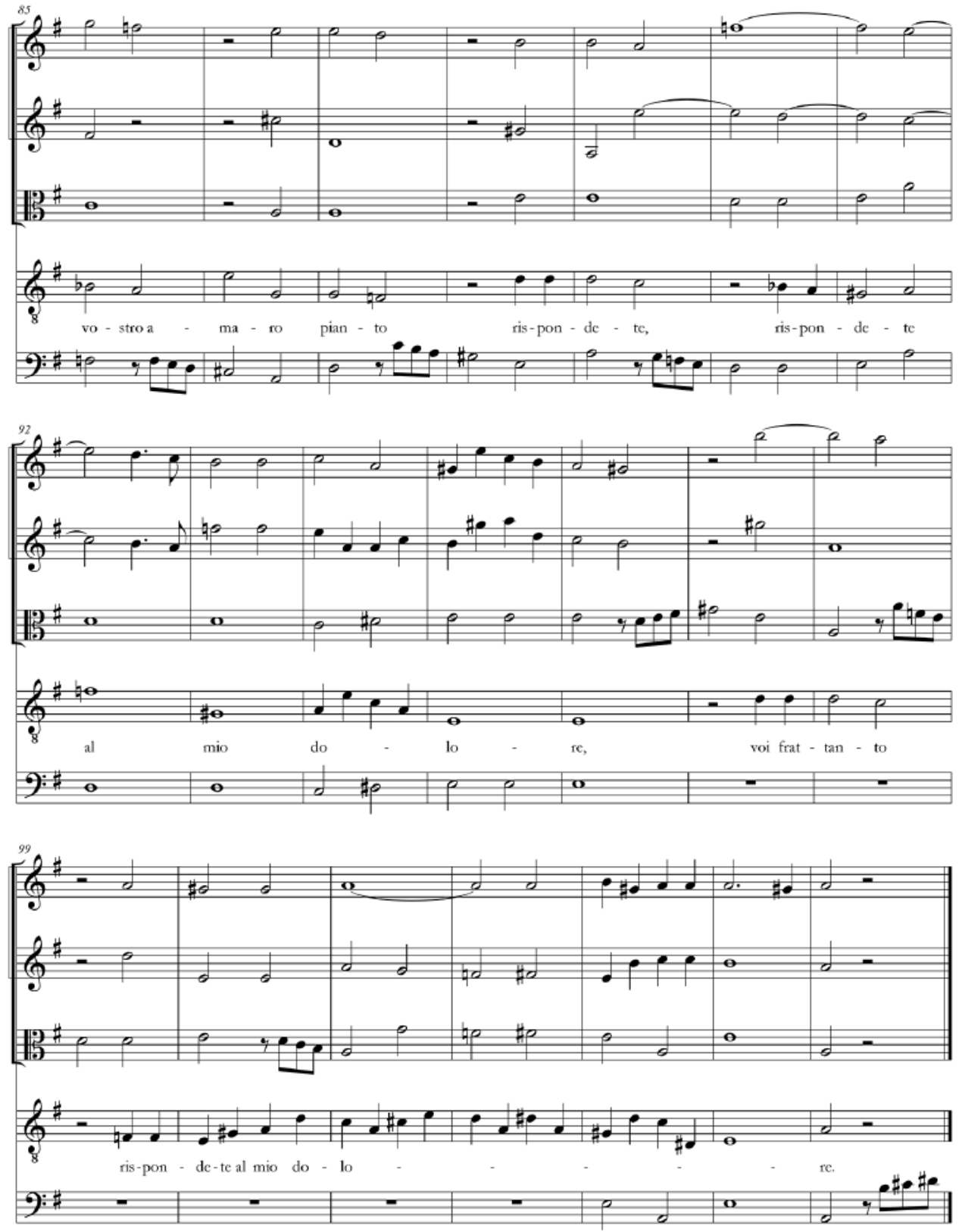

D.C. al Fine 


\section{Anhang 3}
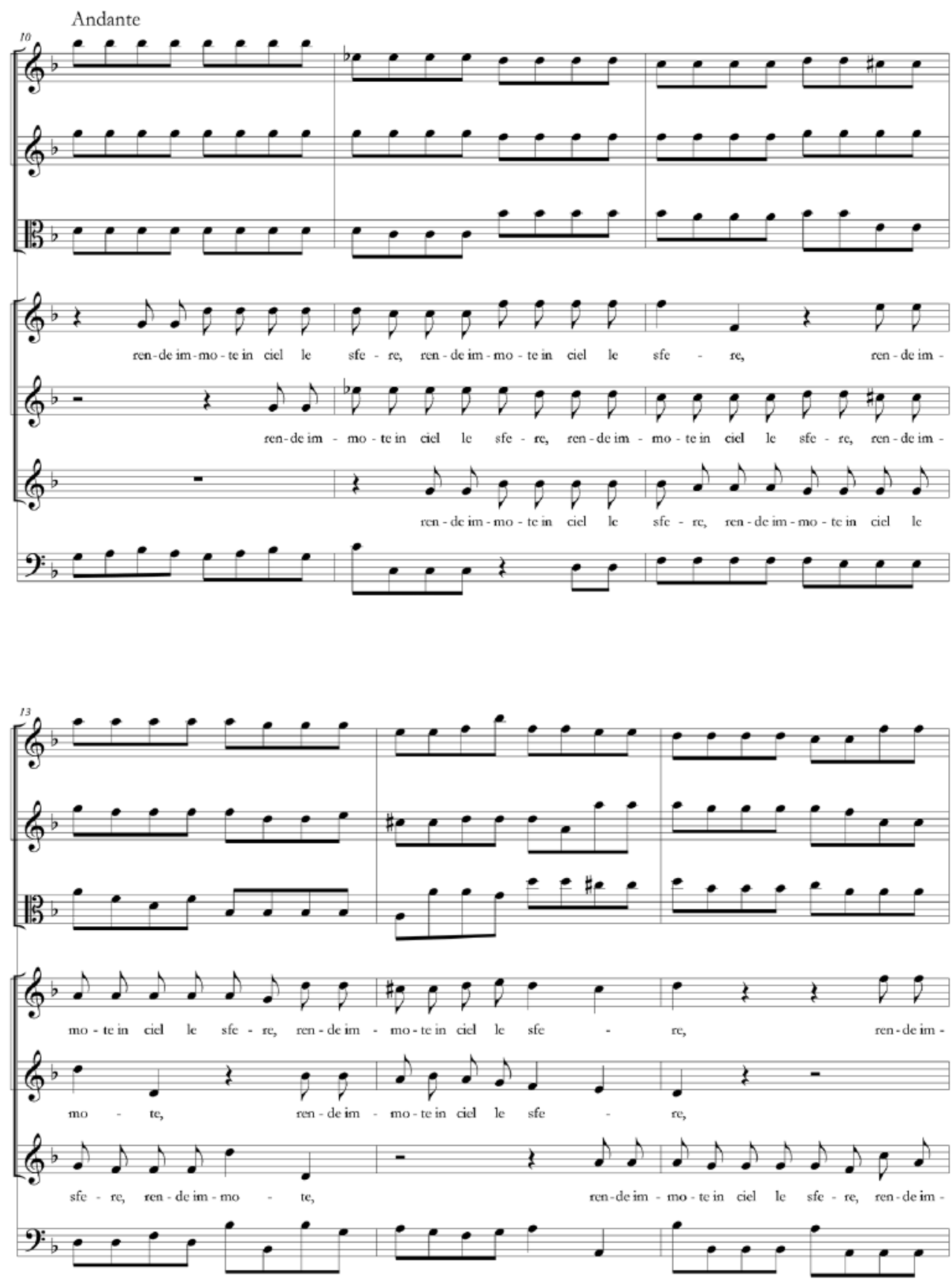

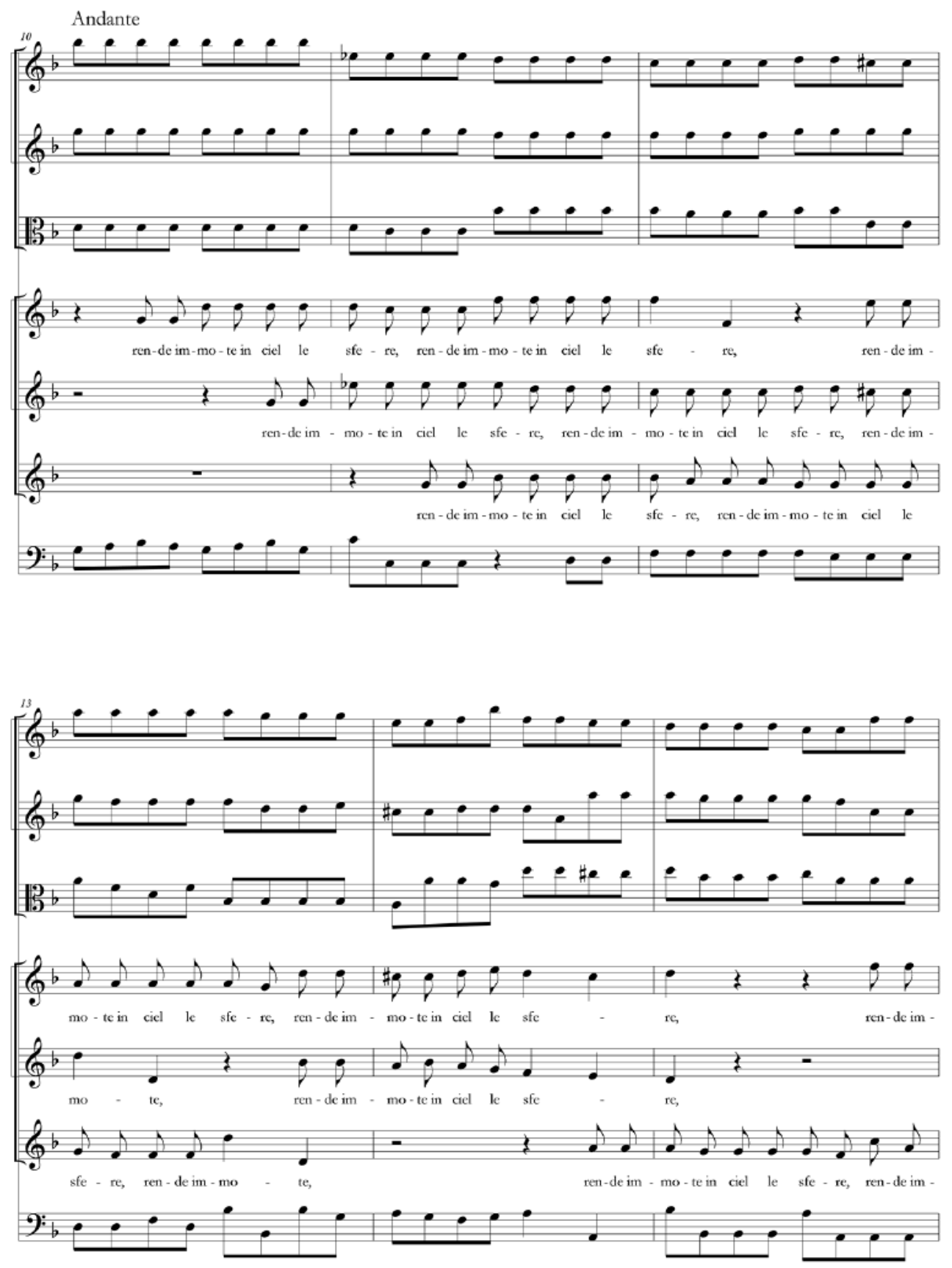


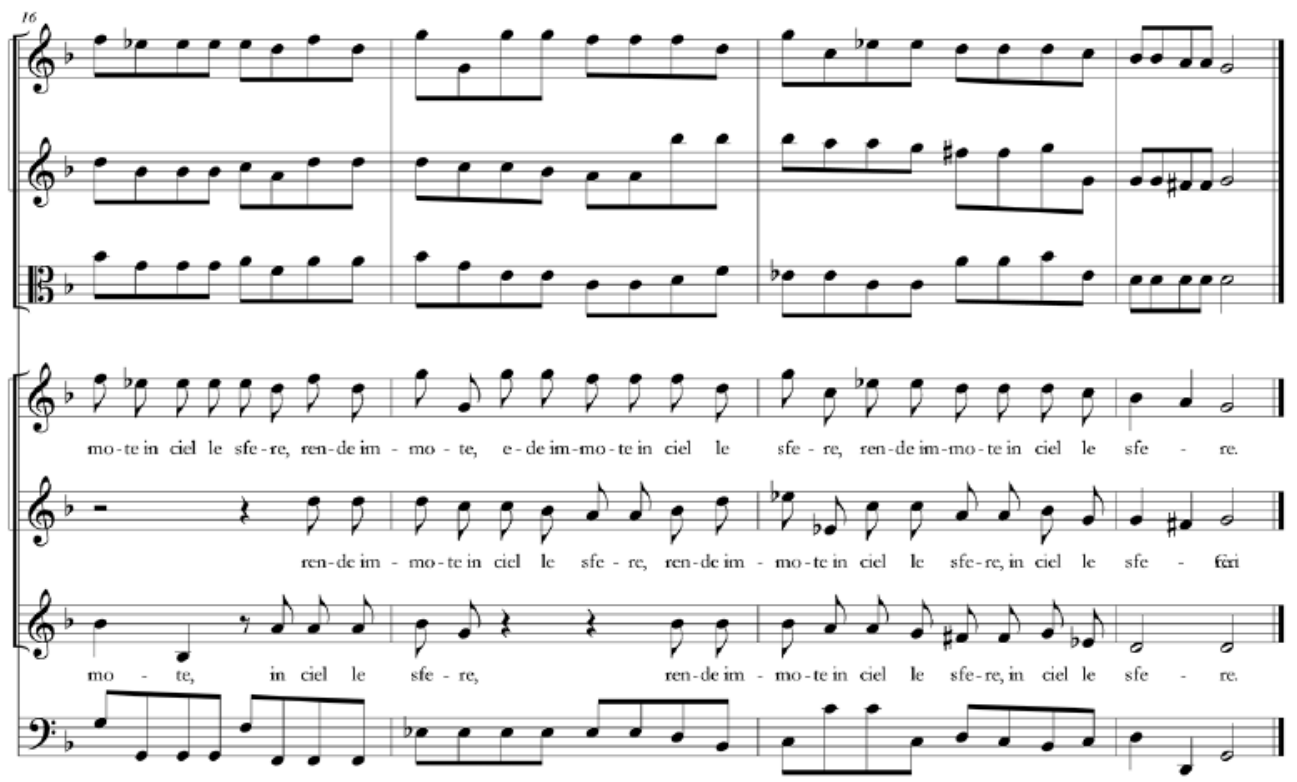

\section{Bibliography}

KAMIEŃSKI, Łucjan. Die Oratorien von Johann Adolf Hasse. Leipzig 1912.

KAČIC, Ladislav. Joseph Umstatt (1711-1762) zwischen Barock und Klassik: Bemerkungen zur Stilentwicklung eines mitteleuropäischen Komponisten. In Zur Geschichte und Aufführungspraxis der Musik des 16.-18. Jahrhunderts in Mittel- und Osteuropa. G. Gruber (Ed.). Bratislava, 2013, pp. 201-215.

KOCH, Michael. Die Oratorien Johann Adolf Hasses. Überlieferung und Struktur (= Musikwissenschaftliche Studien 14). Pfaffenweiler 1989.

MENNICKE, Carl. Hasse und die Brüder Graun als Symphoniker: nebst Biographien und thematischen Katalogen. Leipzig 1906.

PEGAH, Rashid-Sascha. Ein Argippo-Pasticcio. Studi vivaldiani 11, 2011, pp. 63-76.

SPÁČILOVÁ, Jana. Die Rezeption der italiensichen Oper am Hofe des Olmützer Bischofs Schrattenbach. In The Eighteenth-Century Italian Opera Seria. Metamorphoses of the Opera in the Imperial Age (= Colloquium Musicologicum Brunense 42: 2007). Eds. Petr Macek and Jana Perutková. Praha 2013, pp. 75-88.

SPÁČILOVÁ, Jana. Unbekannte Brünner Oratorien neapolitanischer Komponisten vor 1740. Musicologica Brunensia 49, 2014, iss. 1, pp. 137-161.

STROHM, Reinhard. Italienische Opernarien des frühen Settecento (1720-1730). vol. II: Notenbeispiele und Verzeichnisse. Köln 1976.

STROHM, Reinhard. Argippo in „Germania“. Studi vivaldiani 8, 2008, pp. 111-126.

WEILEN, Alexander von. Zur Wiener Theatergeschichte. Die vom Jahre 1629 bis zum Jahre 1740 am

Wiener Hofe zur Aufführung gelangten Werke theatralischen Charakters und Oratorien, Wien 1901. 
